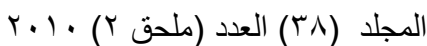
(ISSN 1815- 316X)
مجلة زراعة الر افدين

تقويم حساسية ـ ـ خطوط نقية من الطماطة غير محدودة النمو في انماط زراعية مختلفة للسيطرة على المي

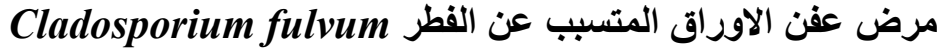

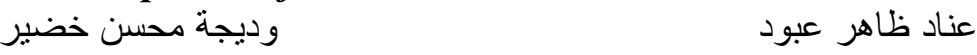 \\ قسم وقاية النبات / كلية الزر اعة /جامعة بغداد
}

\section{الخلاصة}

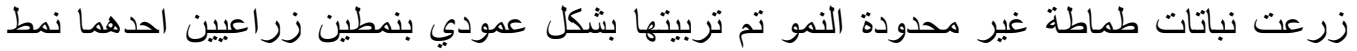

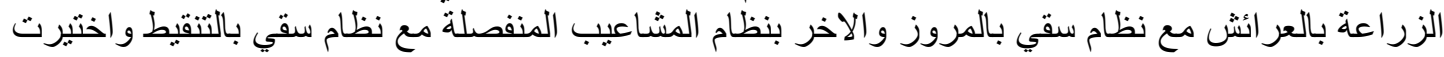

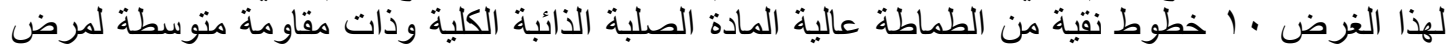
عفن اوراق الطماطة المتسبب عنه الفطر Cladosporium fulvum , وكان الصنف الهجين Cloudia

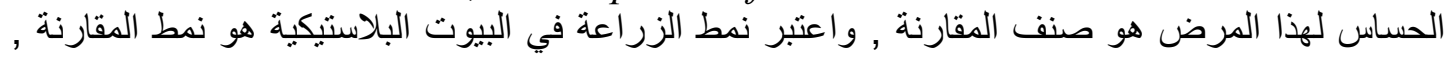

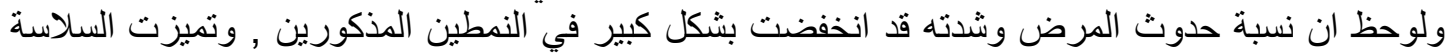

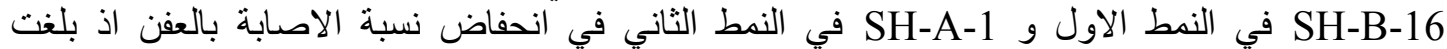

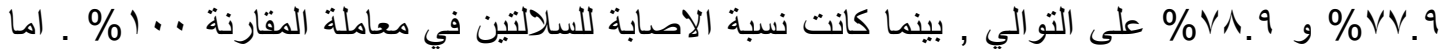

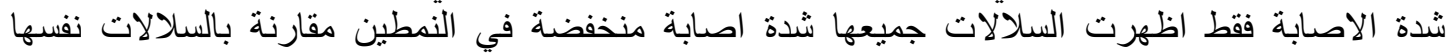
المزروعة في البيوت البلاستيكية اذ لم تتجاوز شدة الاصابة ^^\% في النمطين بينما وصلت شدة الاصابة فيها

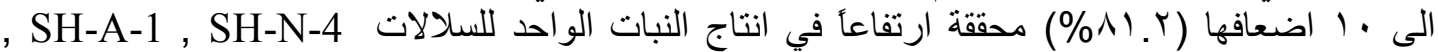
SH-R-61 وSH-B-8 , SH-Q-19 , SH-N-22

البيوت البلاستيكية .

\section{المقدمة}

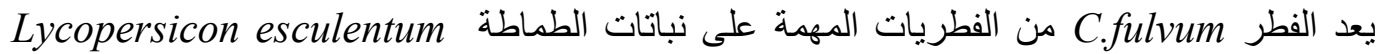

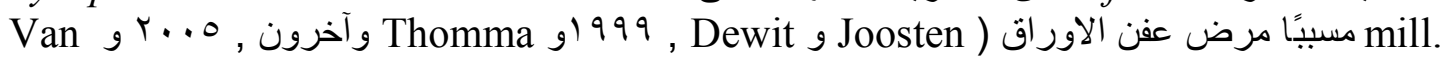

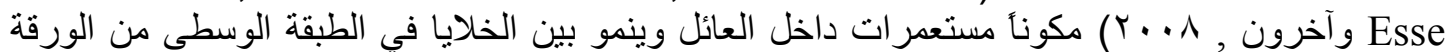

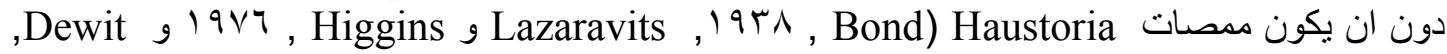

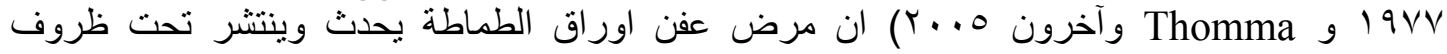

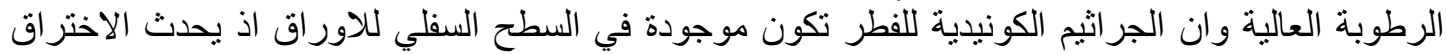

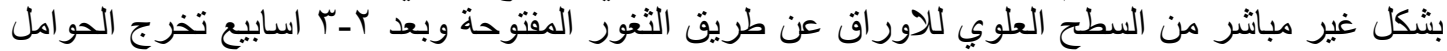

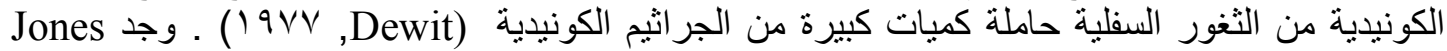

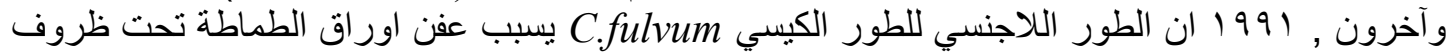

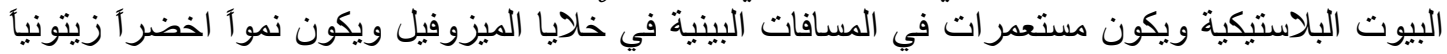

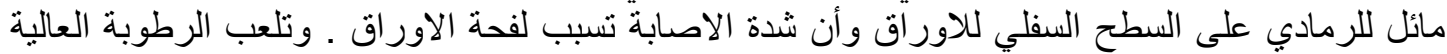

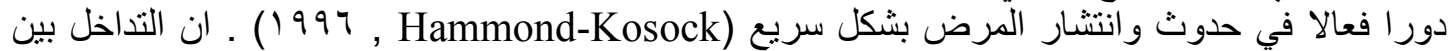

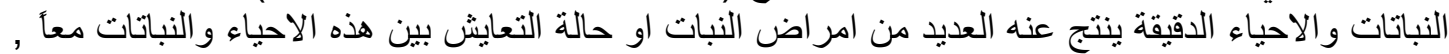

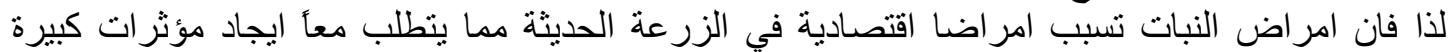

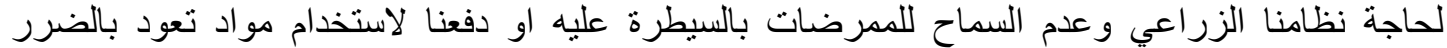

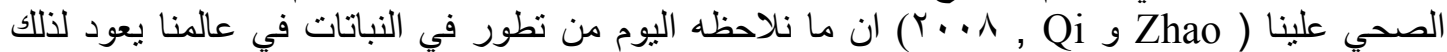

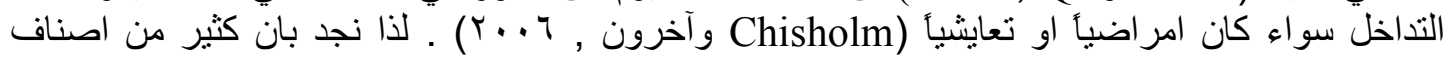

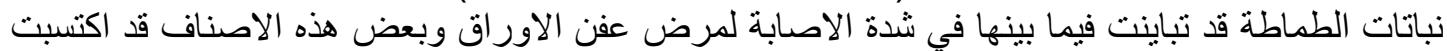

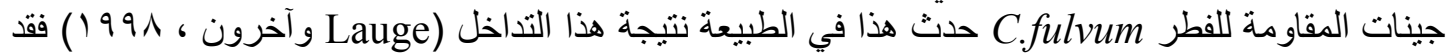

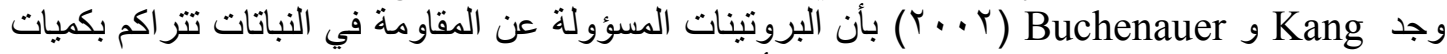

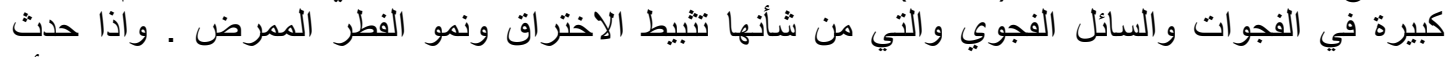

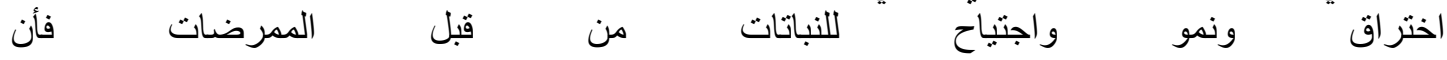

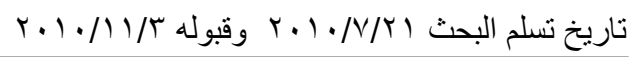


ذلك يعتمد على حقيقيتين اساسيتين هما : 1 - منع النباتات من تمييز الممرضات حتى لا تكون بروتينات

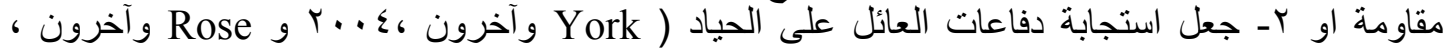

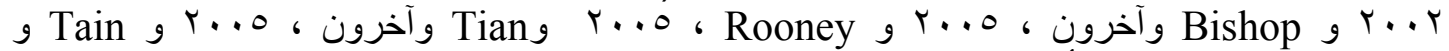

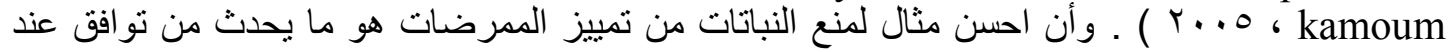

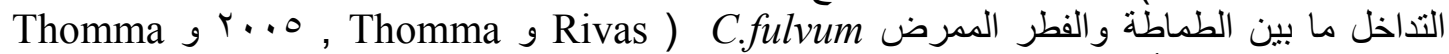

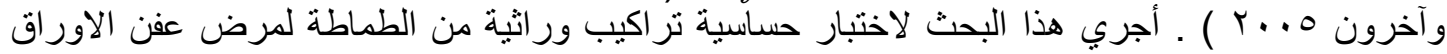

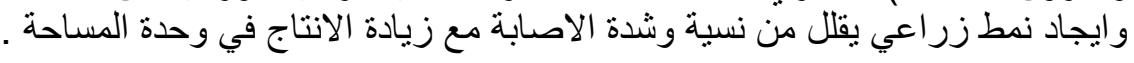

\section{مواد البحث وطر ائقه}

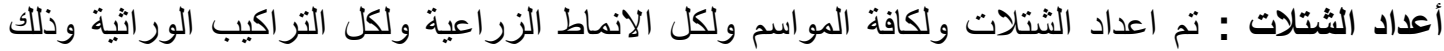

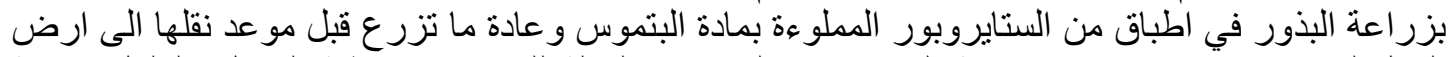

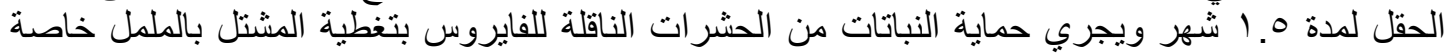

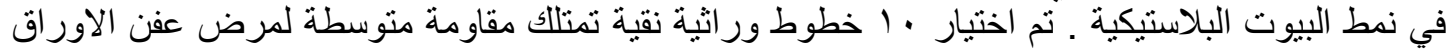

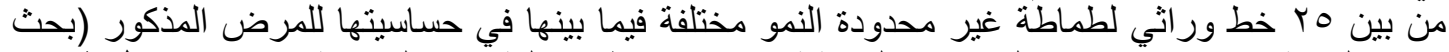

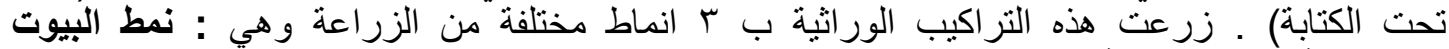

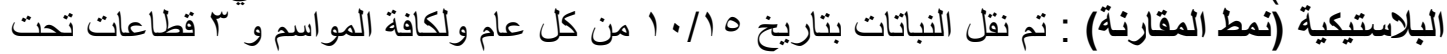

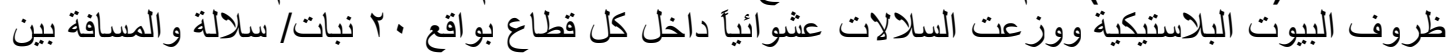

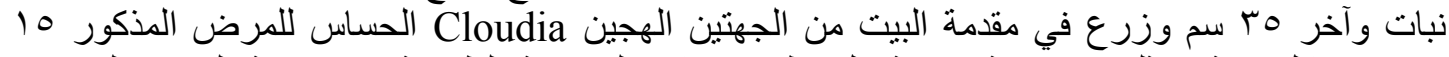

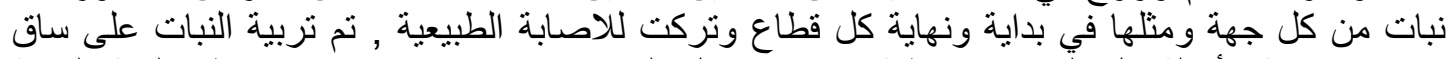

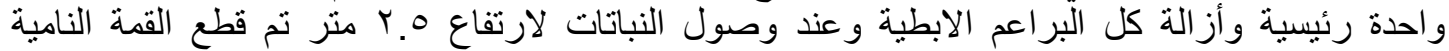

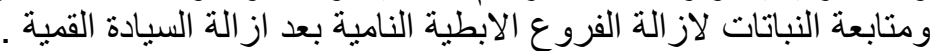

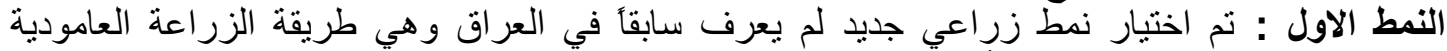

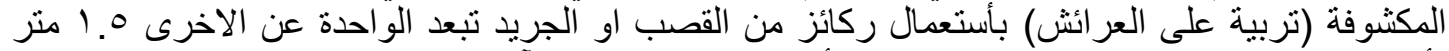

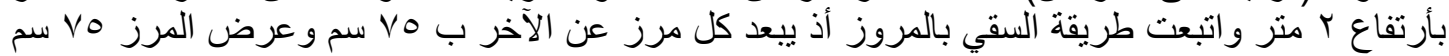

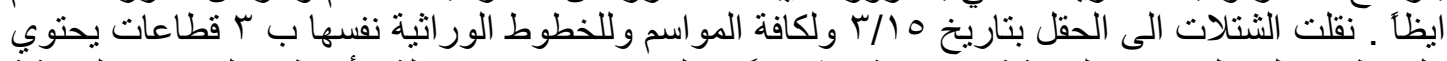

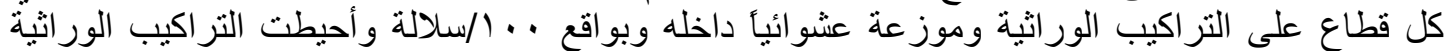

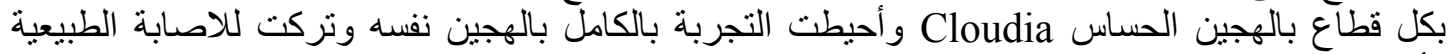

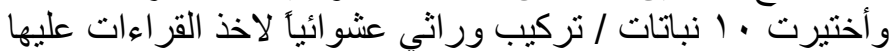

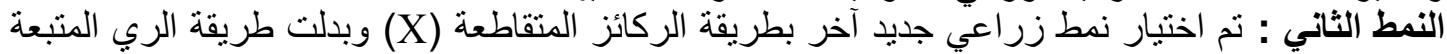

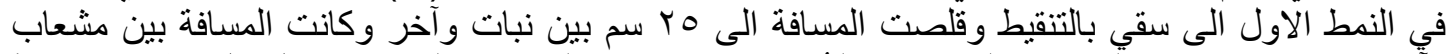

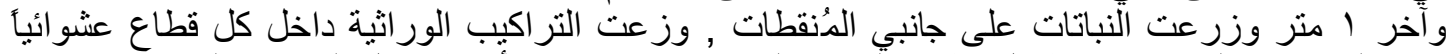

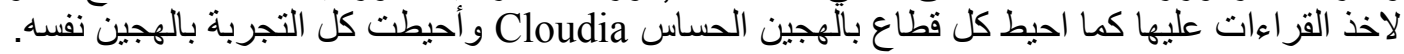

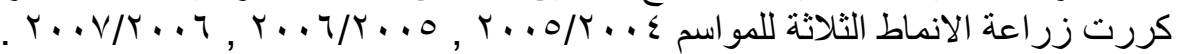

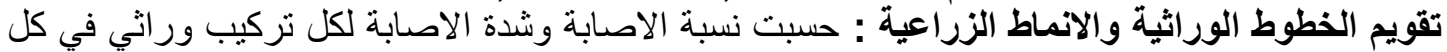

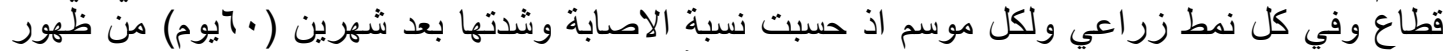

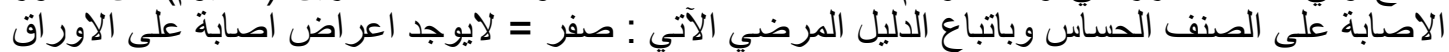

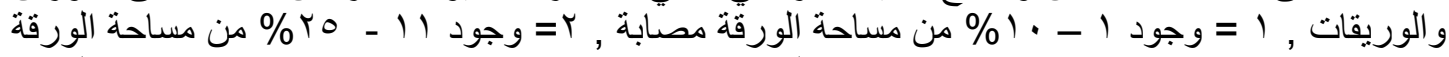

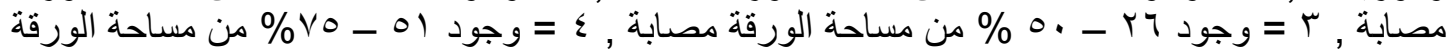

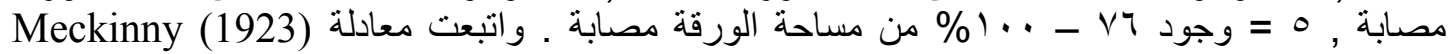

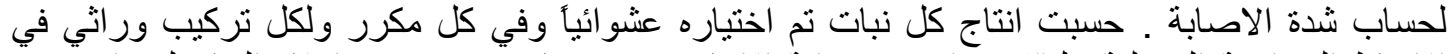

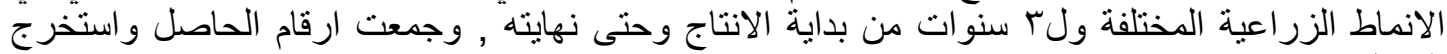

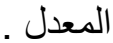
التحليل الاحصائي : حللت النتائج على اساس القطاعات العشو ائية / تجربة عاملية واختبرت المتوسطات

\section{النتائج والمناقشة}

بطريقة (L.S.D) بلانيل

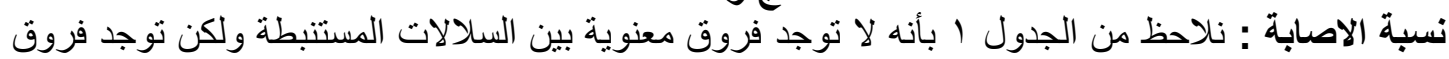

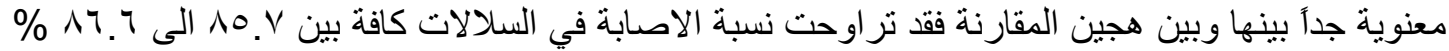

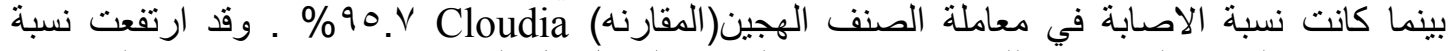
الاصابة في البيوت البلاستيكية للسلالات وصنف المقارنة لتشمل كل النباتات بينما انخفضت هذه النهات النسبة في النيات 


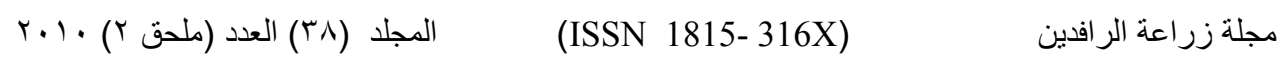

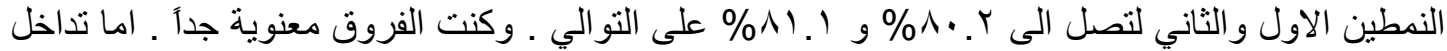

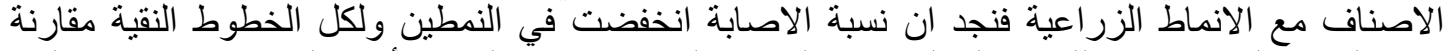

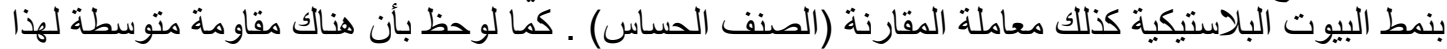

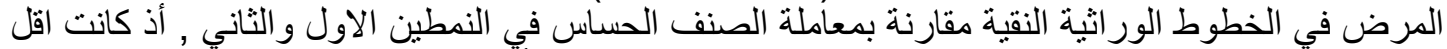

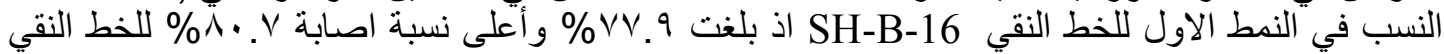

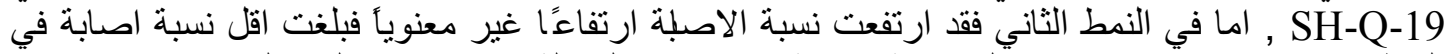

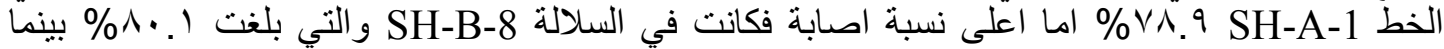

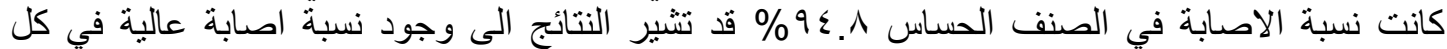

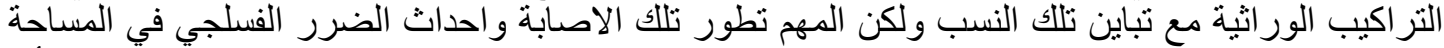

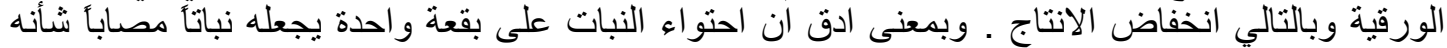

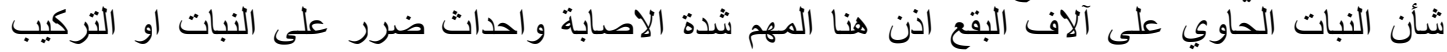

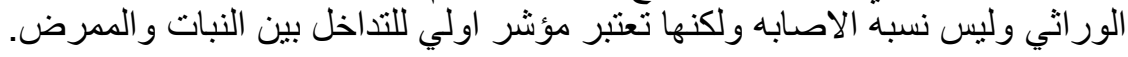

الجدول ( (): نسبة الاصـابة بعفن اوراق الطماطة المتسبب عن الفطر C.fulvum في التُر اكيب الور ائبة للانماط الزر اعية المختلفة

\begin{tabular}{|c|c|c|c|c|}
\hline المتوسطات & النمط الثاني ** & النمط الاول* & البيوت البلاستيكية & الخطوط النقية \\
\hline 10.1 & $V q . Y$ & $V \wedge . Y$ & $1 \ldots$ & SH-N-4 \\
\hline 1.. 1 & $\vee \wedge .9$ & $V 9.5$ & $1 \ldots$ & SH-A-1 \\
\hline ᄉ५. $\varepsilon$ & $\Lambda \cdot . r$ & $V q . Y$ & $1 \ldots$ & SH-N-22 \\
\hline ᄉ५. & $\Lambda \cdot . \cdot$ & $V V .9$ & $1 \ldots$ & SH-B-16 \\
\hline 10.V & $\mathrm{Vq.}$ & V^. 1 & $1 \ldots$ & SH-D-4 \\
\hline 17.9 & $\Lambda \cdot . \cdot$ & $\Lambda \cdot . V$ & $1 \cdots$ & SH-Q-19 \\
\hline 17.0 & $\wedge \cdot .1$ & V9.5 & $1 \cdots$ & SH-P-8 \\
\hline ᄉ५. $\mathrm{V}$ & $\Lambda \cdot . \cdot$ & $\Lambda \cdot . \cdot$ & $1 \ldots$ & SH-H-3 \\
\hline ᄉт.1 & $\vee 9 . \wedge$ & $\nabla \wedge . Y$ & $1 \ldots$ & SH-R-61 \\
\hline A५. $\varepsilon$ & $\wedge .$. & $v 9.1$ & $1 \ldots$ & SH-C-12 \\
\hline $90 . V$ & $9 \varepsilon . \wedge$ & $94 . r$ & $1 \ldots$ & Cloudia المقارنة \\
\hline$r . P T$ & \multicolumn{3}{|c|}{3.434} & L.S.D 0.01 \\
\hline & 11.1 & $\Lambda \cdot . r$ & $1 \ldots$ & المتوسطات \\
\hline & \multicolumn{3}{|c|}{1.1 .1} & L.S.D 0.01 \\
\hline
\end{tabular}

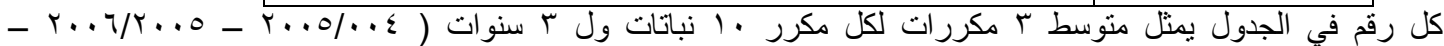

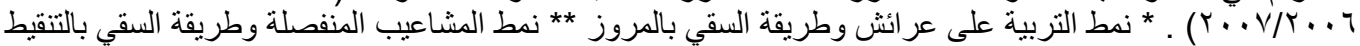

شدة الاصابة بالممرض : يتضح من الجدول r ان الخطوط الوراثية قد تمزيت بانخفاض شدة الاصابة

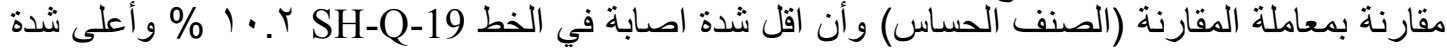
اصفابة كانت في الخط الوراثي

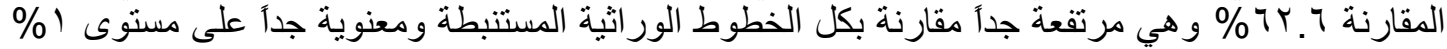

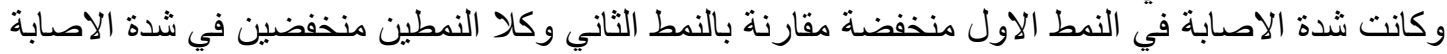

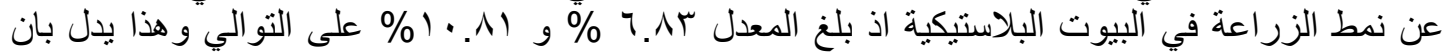

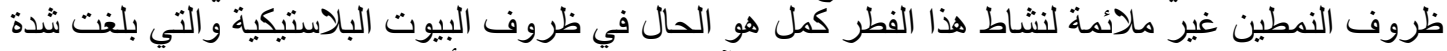

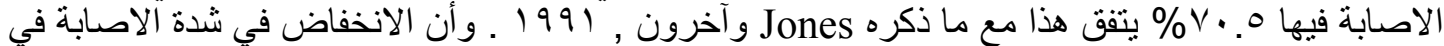

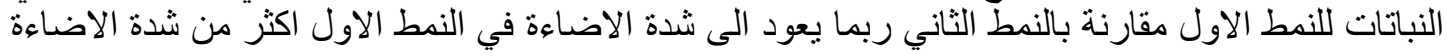

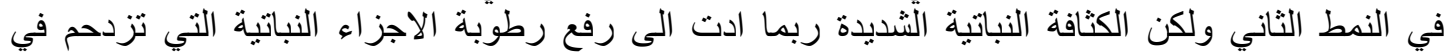

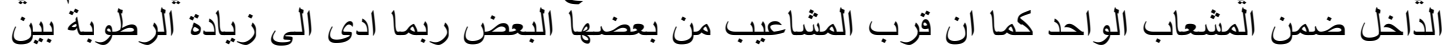

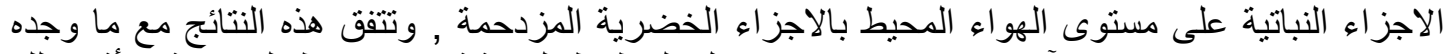
Hommond - Kosack

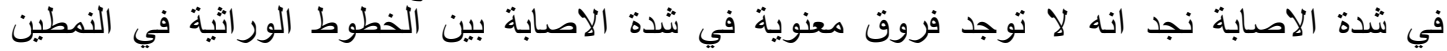

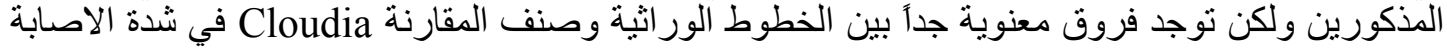




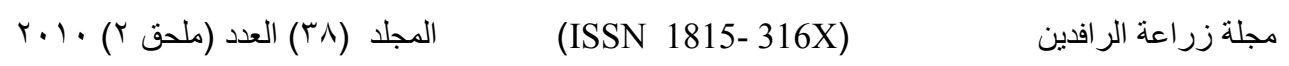

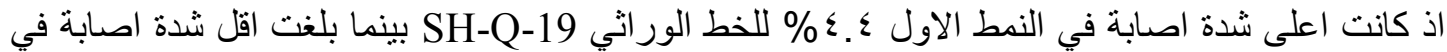

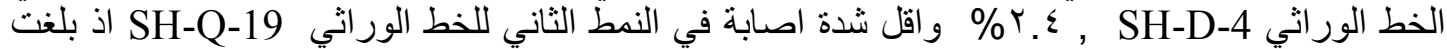

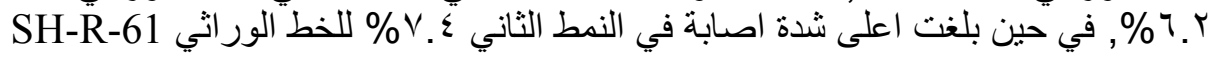

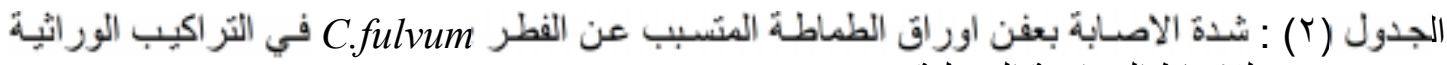
ل اللانماط الزر اعية المختلفة.

\begin{tabular}{|c|c|c|c|c|}
\hline المتوسطات & النمط الثاني & النمط الاول & البيوت البلاستيكية & الخطوط النقية \\
\hline rq. & 7.1 & $\varepsilon . r$ & VI.V & SH-N-4 \\
\hline rq. & V.r & T. & $V V_{.1}$ & SH-A-1 \\
\hline$\Gamma \cdot . \Sigma$ & 7.1 & $\varepsilon . r$ & $\Lambda \cdot .1$ & SH-N-22 \\
\hline$r \cdot . \Sigma$ & V.I & r.A & $\Lambda \cdot . r$ & SH-B-16 \\
\hline rq.V & 7.0 & T.z & $\Lambda \cdot .1$ & SH-D-4 \\
\hline $1 \cdot . r$ & $7 . Y$ & $\varepsilon . \varepsilon$ & 19.9 & SH-Q-19 \\
\hline$\mu \cdot r$ & 7.1 & r.s & $1 \cdot .9$ & SH-P-8 \\
\hline$r \cdot . \Sigma$ & 7.1 & r. & $1 \cdot .9$ & SH-H-3 \\
\hline $1 \cdot . V$ & $V . \varepsilon$ & $r .7$ & YI.. & SH-R-61 \\
\hline$r \cdot .0$ & V.. & $r . r$ & $\Lambda 1 . r$ & SH-C-12 \\
\hline 74.7 & $0 . .1$ & $\varepsilon \cdot .1$ & $9 V .5$ & المقارنةCloudia \\
\hline$r .+1 r$ & \multicolumn{3}{|c|}{0.119} & L.S.D 0.01 \\
\hline & $1 \cdot .11$ & I. & $V \cdot .0$ & المتوسطات \\
\hline & \multicolumn{3}{|c|}{ 1.OT } & L.S.D 0.01 \\
\hline
\end{tabular}

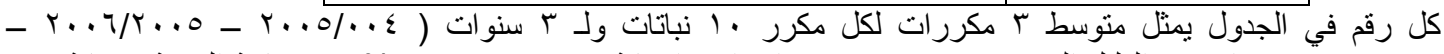

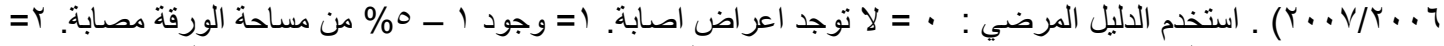

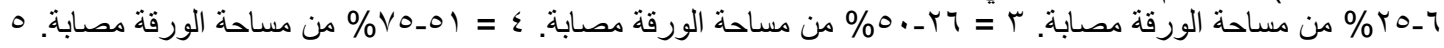

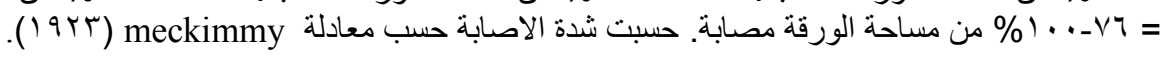

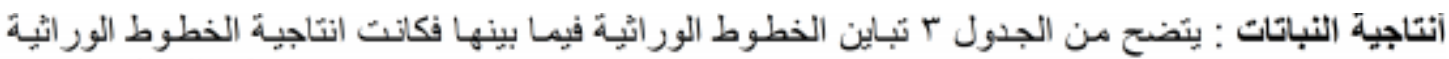
SH-B-8, SH-R-61 , SH-N-4, SH-2-N-22 , SH-Q-19

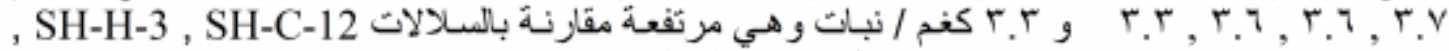

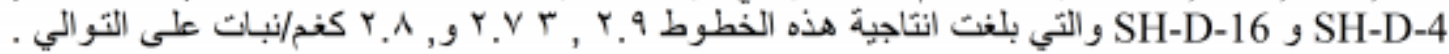

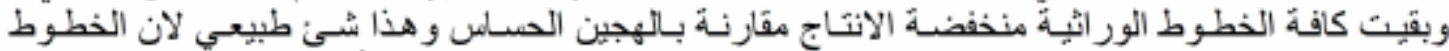

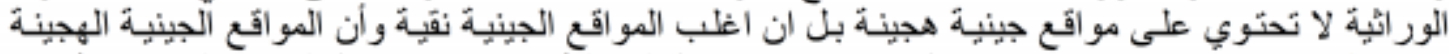

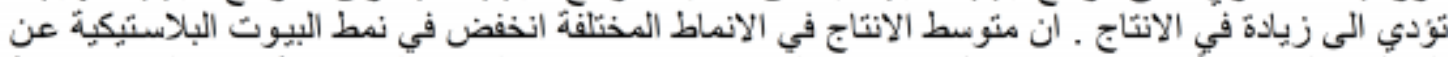

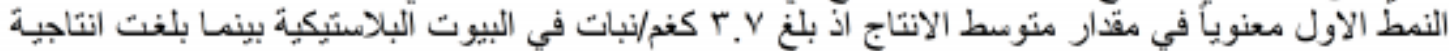

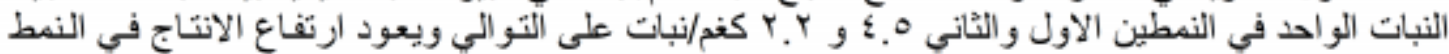

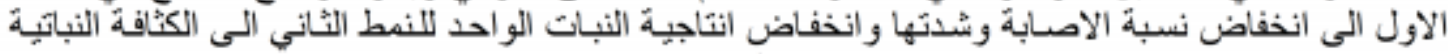

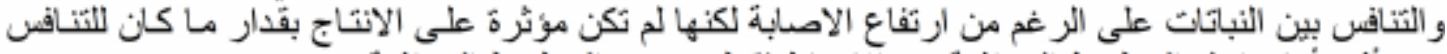

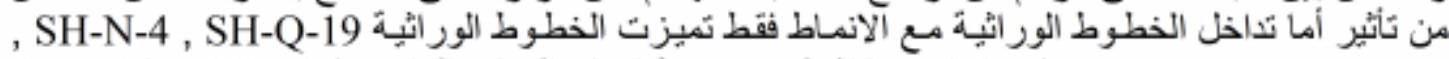

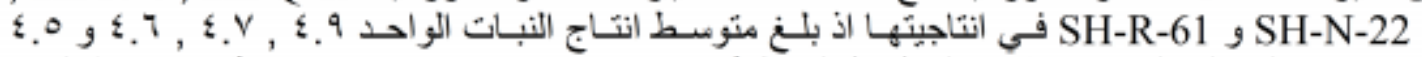

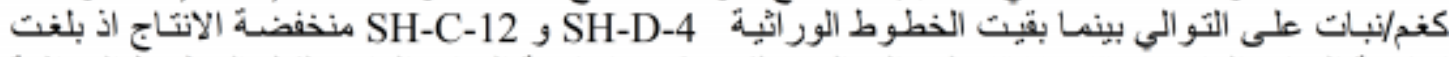

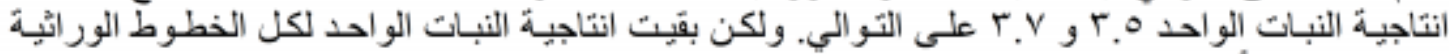

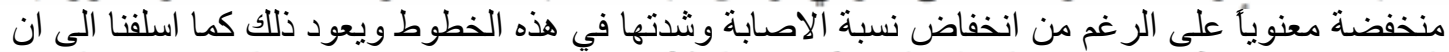

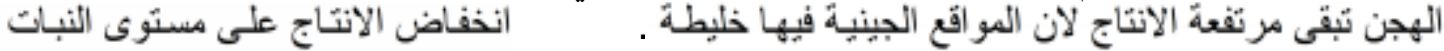

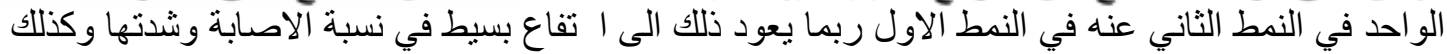

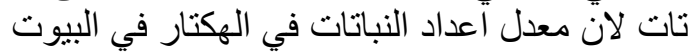

( ) : معدل انتاجية النبات الواحد ( ) للتر اكيب الور اثية في الانماط الزر اعية المختلفة. 


مجلة زراعة الر افدين $\quad$ ( ) $\quad$ ( ) $\quad$ (ISSN 1815-316X) $\quad$ ( )

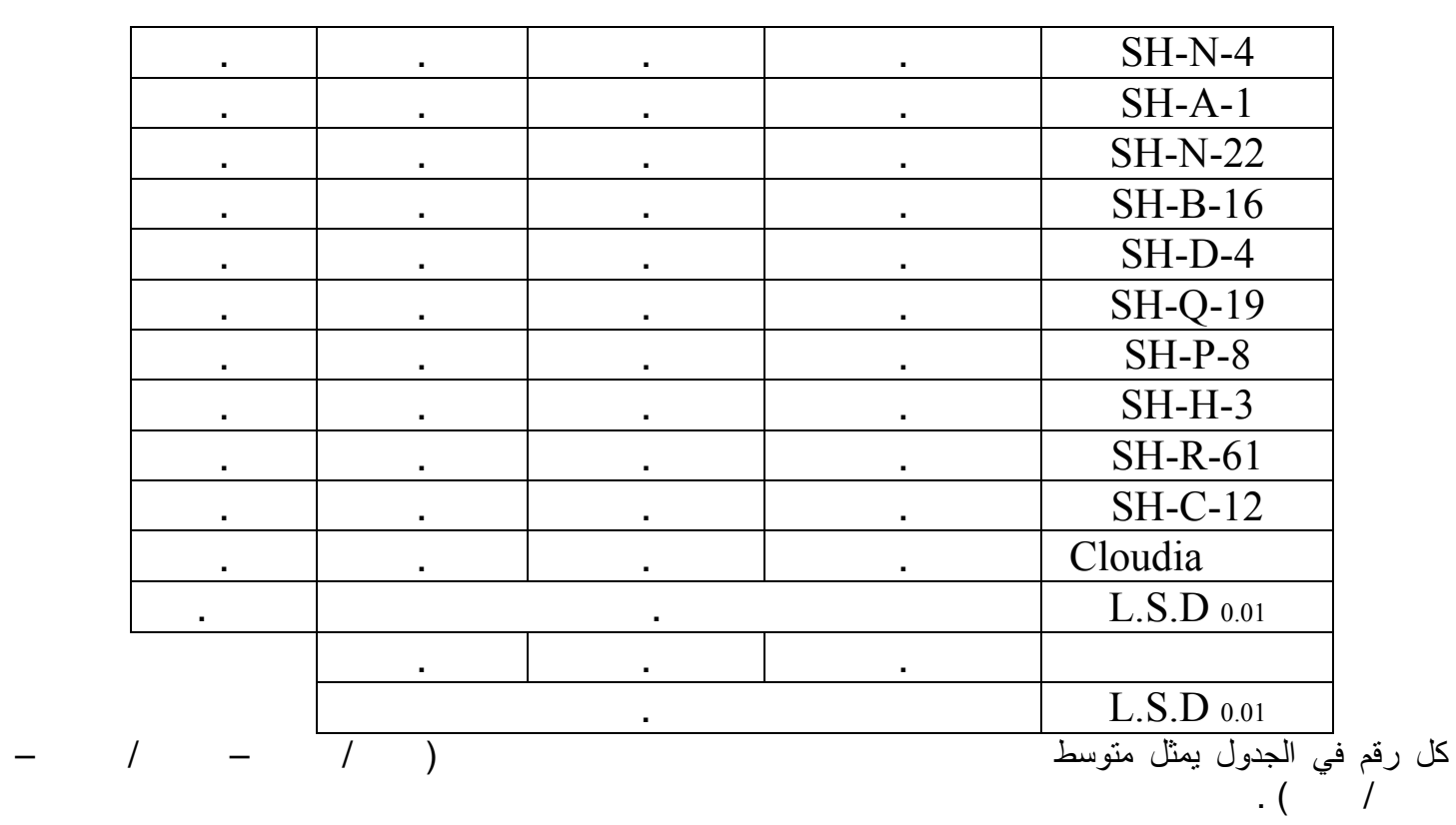

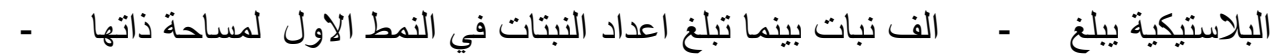

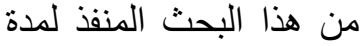

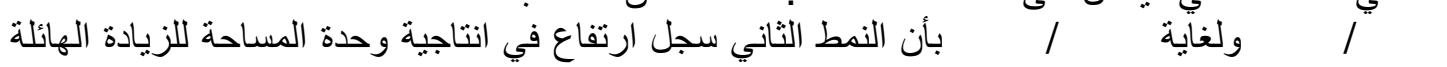

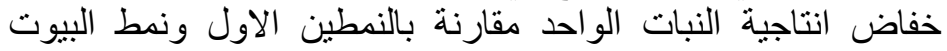

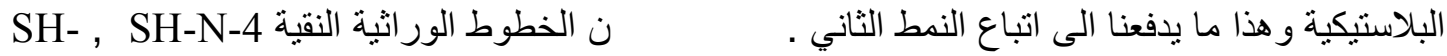
SH-R-61 SH-Q-19 , N-22

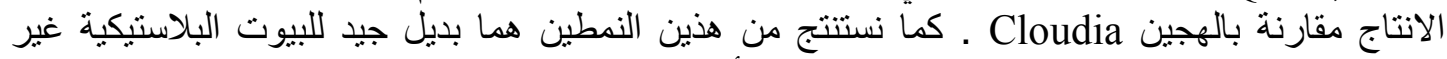

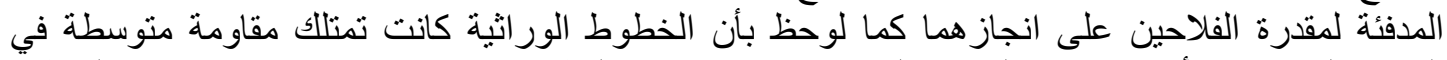
البيوت البلاستيكية أزدادت هذه الصفة بالهروب من الاصنابة بالفطر C.fulvum عندما زر عت في النمطين المذكورين.

\title{
EVALUATION OF SENSITIVITY FOR 10 PURELINES TOMATOES INDETERMINATE IN DIFFERENT AGRICULTURAL MODES TO CONTROL OF LEAF MOLD CAUSED BY Cladosporium fulvum
} Inad D. Abood Wadeja M. Khuther

Plant Protection Dept.College of Agric. Univ. of Baghdad

\begin{abstract}
Indeterminate tomato pure lines were planting in two agricultural modes :mode of arbour and seprate drip irrigation and selective 10 purelines highly total soluble solvent and have moderately resistance for leaf mold caused by Cladosporium fulvum. Claudia hybrid was sensitive for leaf mold disease as cultivar control treatment and the plastic houses as acontrol mode. The percentage of disease incedent and severity were decreased in new agricultural modes, the pureline : SHB-16 in the first mode and SH-A-1 in the second mode were decreased of percentage disease incedent $77.9 \%, 78.9 \%$ respectively, while in conyrol treatment reached to $100 \%$. All the purelines were decreased of severity in two agricultural modes and not exceeded (8\%) when compared with same purelines which planting in plastic house mode which reached disease severity to ten time $(81.2 \%)$ and the
\end{abstract}


production of each plant was increased for the purelines SH-N-4, SH-A-1, SH-N-22 , SH-Q-19, SH-P-8 , SH-R-61 in new two agricultural modes when compared the same purelines production in plastic house mode

Arie, t., H.Takahashi, M. Kodama and T. Teraoka (2007). Tomato as a model plant for plant-pathogen interactions. Plant Biotechnol. 24: 135-147.

Bishop, J.G., D.E.H.: poll, s. Bashir, C.M Damascene, J.D. Seeds, and J.K. Rose (2005). Welection on Glycine beta- 1,3 -endoglucanasegenes differentially inhibited by a Phytophthora glucanase inhibited protein. Genetics. p: 10091019.

Bond, T.E T.(1983). infection experiments with Cladosporium fulvum cooks and related species. Ann. App. Biol. 25. 277-307.

Booney, H.C. and J.W. Vant Klooster, R.A. Van der Hoorn, M.H., a.j. jouster, J.D.G . Jones, and P.J.G.M De Wit (2005). Cladosporium Avr2 Inhibit tomato Rec 3 protease required for $\mathrm{Cf}$-2-deperdent disease resistance. Science. 308:1783 - 1986.

Chisholm, S.T.,G. Coaker ,B. Day and B.J.Staskawicz (2006). Host - microbe interaction: shaping the evaluation of the plant immune response. Cell. 124:803-814.

De Wit, P.J.G.M. (1977).A light and scaling - electron microscopic study ofinhibition of tomato Plants by virulent and a virulent races of Cladosporium fulvum. Neth. J. plant pathol. 83:109-122.

Hammond-kosack, K. E., P. Silverman; I. Paskin and J. D. G. jones (1996). Race specific elicitors of Cladosporium fulvum indice changes cell morphology and the synthesis of ethylene and salicylic acid in tomato plant carrying the corresponding of disease resistance gene . Plant physiol. 110 : 1381-1394.

Hammond-kosack, K.E. and J.D.G. Jones (1994). Incomplete dominance of tomato $\mathrm{Cf}$ genes for resistance to Cladosporium fulvum. Mol. Plant Microbe Instract. 7:58-70.

Jones, J.B.; J.P. jones, R.E. Stall and T.A. Zitter (1991). Compendium of tomato disease . APS Press, st. paul , mn, USA. PP:100.

Joosten, H.H.A.J. and P.J. G.M. de Wit (1999). The tomato Cladosporium fulvum interaction. Aversatile expermintal system to study plant pathogen interaction. Annu. Rev. Phytopathoil. 37: 335- 367.

Kang, Z.; and H. Buchenaner (2002). Immunocyfo chemical localization of beta 1,3 - gluconase and chitinase in Fusarium culmorum infected wheat spikes. Physiol. Mol. Plant Pathol. 60: 141-153.

Lauge, R., A. P. Dmitriev, M.H.A.J. Joosten, and P.J.G.M. De Wit (1998). ddition of resistance gene (s) against Cladosporium fulvum present on the Cf- 9 intogression segment are associated with strong PR protein accumulation. M.decmber plant- microbe interation. 11: 301-308.

Lazarovits , G. and V.J. Higgins (1976). Histological comparison of Cladosporium fulvum race 1 on immune resistant and susceptible tomato varieties. Can.J.Bot. 54: 224-234.

Rivas, s. and C.M. Thomas (2005). Molecular interactions between tomato and the leaf mold pathogen Cladosporium fulvum. Annu. Rev. Phytopathol. 34: 395-436. 
Rose, J.K.C., K.S. Ham, A.G. Darvill, and p. Albersheim (2002). Molecular cloning and characterization of slucanase inhibitor protein: coevolution of a counter defense mechanism by plant pathogens. Plant Cell. 14: 1329-1345.

Thomma , B.P. H.J., H.P. Van Esse , P.W. Crons and P.J.G.M. De Wit (2005). Cladosporium fulvum (Syn Passalora fulva), a highly specialized plant pathogen as a model for functional studies one plant pathogenic Mycoshaerellaceae . Mol. Plant Pathol. 6:379-393.

Tian, M., and S. Kamoun (2005). A two disulfids bridge kazal domain from phytophthora exhibits stable inhibitory activity against serine proteases of inhibition fumly BMC. Biochem. 6:15.

Tian, M., B. Benedetti and S. Kamoun (2005). A second kazeol-like protiens inhibitor from phytophthora infestans inhibits and intaracts with the apoplastic pathogensis related protease P69 B of tomato . Plant physiol. 138. 1785-1793.

Tian, M., E. Huitoma , L.DaCunha, T. Torlo-Alalibo , and S. Kamoun (2005). A kazal-like extra cellulose serinc protease inhibitor from Phytophthora infestans forysts the tomato pathogenesis related proteins P69 B. J. Biol. Chem. 279. $26370-26377$.

Van Esse, H.p., J.W. Thomma (2008). The Cladosporium fulvum. And virulence protein Avr2 inhibits host proteases required for based defense. The plant cell. 20: 1948-1963.

Wit P.J.G.M. and G.spikman (1982). Evidence for the occurrence of race and cultivar - specific elicitors of necrosis in intracellular fluid of compatible interaction of Cladosporium fulvum and tomato. Physiol. Plant Patho. 21:1-11.

York, W.S., Q. Qin, and J.K Rose (2004). Protinaceous inhibitors of endo-bests glucanases . Biochim. Biophags. Acts . 1696 . 223- 233.

Zhao, s. and x. Qi (2008). Signaling in plant disease resistance and symbiosis. J. Integr. Plant Biol. 20. 799-807. 